University of Wollongong

Research Online

Faculty of Informatics - Papers (Archive)

Faculty of Engineering and Information

Sciences

August 2004

\title{
Distributed, collaborative learning environments using ad hoc networks
}

\author{
A. Fuller \\ University of Wollongong, annef@uow.edu.au \\ Penelope McFarlane \\ University of Wollongong, penney@uow.edu.au \\ D. F. Saffioti \\ University of Wollongong,dfs@uow.edu.au
}

Follow this and additional works at: https://ro.uow.edu.au/infopapers

Part of the Physical Sciences and Mathematics Commons

\section{Recommended Citation}

Fuller, A.; McFarlane, Penelope; and Saffioti, D. F.: Distributed, collaborative learning environments using ad hoc networks 2004.

https://ro.uow.edu.au/infopapers/138

Research Online is the open access institutional repository for the University of Wollongong. For further information contact the UOW Library: research-pubs@uow.edu.au 


\title{
Distributed, collaborative learning environments using ad hoc networks
}

\author{
Abstract \\ Computer assisted distributed or collaborative learning environments have been in existence for some \\ time, however one impediment to more widespread use has been the need for a permanent wired network \\ infrastructure. In this paper we discuss the development of a prototype tool that allows the formation of \\ an ad hoc network at any time, and in any locale. \\ Disciplines \\ Physical Sciences and Mathematics \\ Publication Details \\ This article was published as: Fuller, A, McFarlane, P \& Saffioti, D, Distributed, collaborative learning \\ environments using ad hoc networks, Proceedings IEEE International Conference on Advanced Learning \\ Technologies, 30 August-1 September 2004, 705-707. Copyright IEEE 2004.
}




\title{
Distributed, Collaborative Learning Environments Using Ad Hoc Networks
}

\author{
Anne Fuller, Penney McFarlane \& Daniel Saffioti \\ University of Wollongong - Australia
}

\begin{abstract}
.
Computer assisted distributed or collaborative learning environments have been in existence for some time, however one impediment to more widespread use has been the need for a permanent wired network infrastructure. In this paper we discuss the development of a prototype tool that allows the formation of an ad hoc network at any time, and in any locale.
\end{abstract}

\section{Introduction.}

Distributed learning environments (DL) and computer assisted collaborative learning environments (CACL) that allow participants to share information are not new. It is even possible to use a number of simple technologies such as Netmeeting to allow sharing of desktop information between an instructor and a group of students or among the students themselves to create such an environment [1]. However these environments generally require the computers sharing the information to be connected to the internet or to a wired network or be in close proximity to a wired access point. Such approaches limit both the numbers of students and the locations that can be involved. The ability to create new networks as, when and wherever they may be required will, we believe, increase the domains to which DL and CACL may be applied and thus lead to greater deployment of such environments.

In this paper we discuss the development of iBroadcast [2], a prototype of a tool that permits the setting up of ad hoc broadcasts between laptops and personal Digital Assistants (PDAs). We discuss the advantages of ad hoc networking in to both DL and CACL. Finally we describe some related work.

\section{Distributed Learning Environments and iBroadcast.}

A distributed learning environment allows students and educators to interact from a number of different locations. Most interactions in common environments such as WebCT or Blackboard are asynchronous. iBroadcast is a prototype environment that allows students and educators to interact in real time through the sharing of video content ( their interactions on the desktop of a computer), data (files) and discussion (text messaging).

An important feature of this environment is the ability to support bulk communications as illustrated in Figure 1. The sharing of video content is particularly suited for lectures, tutorial or demonstration environments where a central authority i.e. the lecturer assumes control. The sharing of files or messaging supports either one to one or one to many communication. Although the current prototype does not support true collaborative learning in the sense that other users can interact with one another, such support is planned in future versions.

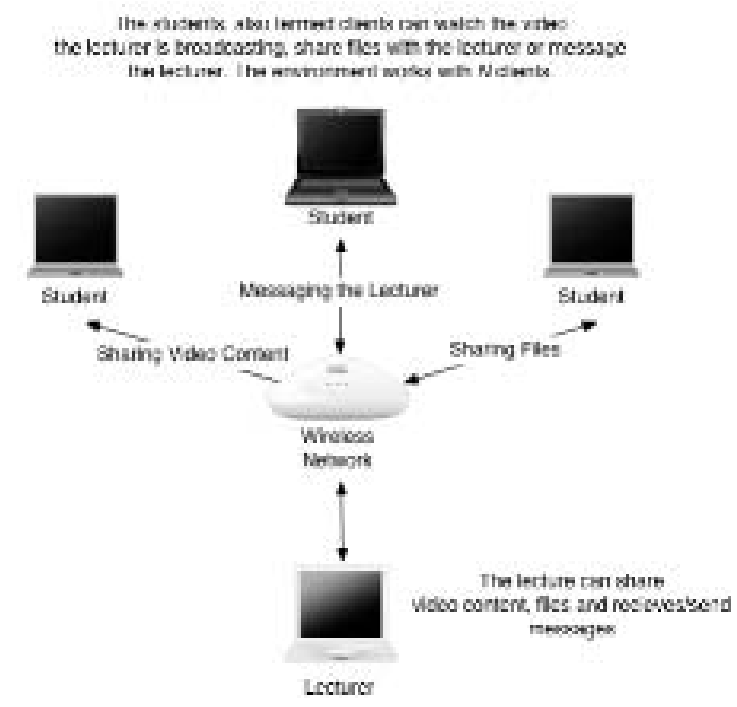

Figure 1: Ibroadcast Communications Model

The most significant difference between iBroadcast and other learning environments is that it is a real time learning technology, which shares data via ad hoc/fixed networks. The software utilizes Apple's Rendezvous [3] plug and play protocol, thus requires no knowledge of networking and requires no administration. This makes the technology particularly suited to small collaborative environments, eg. lectures where knowledge or technical skills cannot be assumed. 


\section{4. iBroadcast Features, Innovations and Requirements.}

iBroadcast supports varying modes of communication and interaction. These features are streaming, file sharing and messaging.

iBroadcast allows real-time streaming of a computer's desktop. The user of the iBroadcast server (usually the instructor) sets the area to be transmitted to the clients (students), running on other personal computers. Once transmission has commenced on the server, clients begin to see the section of the desktop that the server has shared. iBroadcast's video sharing service is unidirectional as seen in Figure 2.

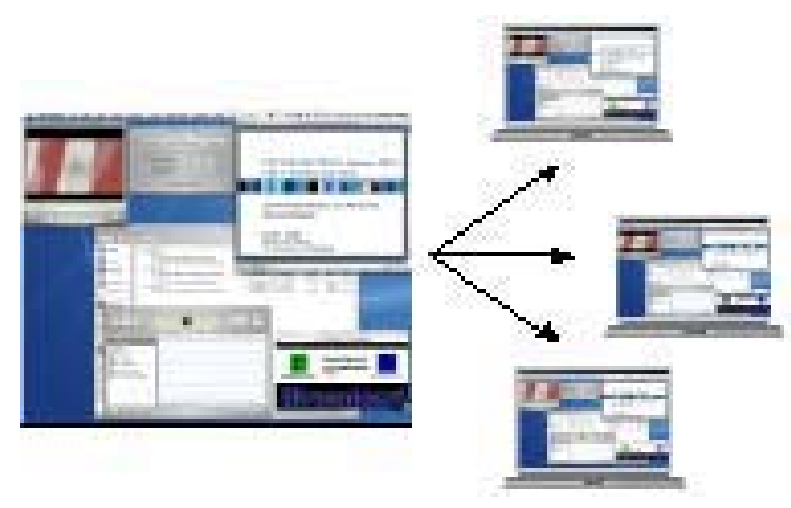

Figure 2: iBroadcast Client/Server features.

With a standard iBroadcast server, the instructor may start a Powerpoint presentation, and then demonstrate with a real example using another application. A student can be sitting inside or outside the lecture theatre yet see everything the lecturer does on their workstation. In addition, the server can record the session to a file, which can be viewed by users at a later time. It is believed that real-time streaming will positively impact on instruction/teaching.

This real-time, wireless effect is achieved using some unique Apple technologies e.g Rendezvous and Quartz [4]. Quartz allows the server to identify which regions of the server's display have changed thus allowing only the transitions to be broadcast. This allows more rapid updating of client screens.

The file sharing feature eliminates the need for setting up file sharing environments. The server's user selects which files they wish to make available to the clients. Clients may then select from the list of files available and download when desired. A potential application for this may be in a lecture where a lecturer wishes to disseminate a file containing a solution to a problem. This again can be done in real-time. Figure 3 shows how simple it is to use file sharing from both client/server perspectives.

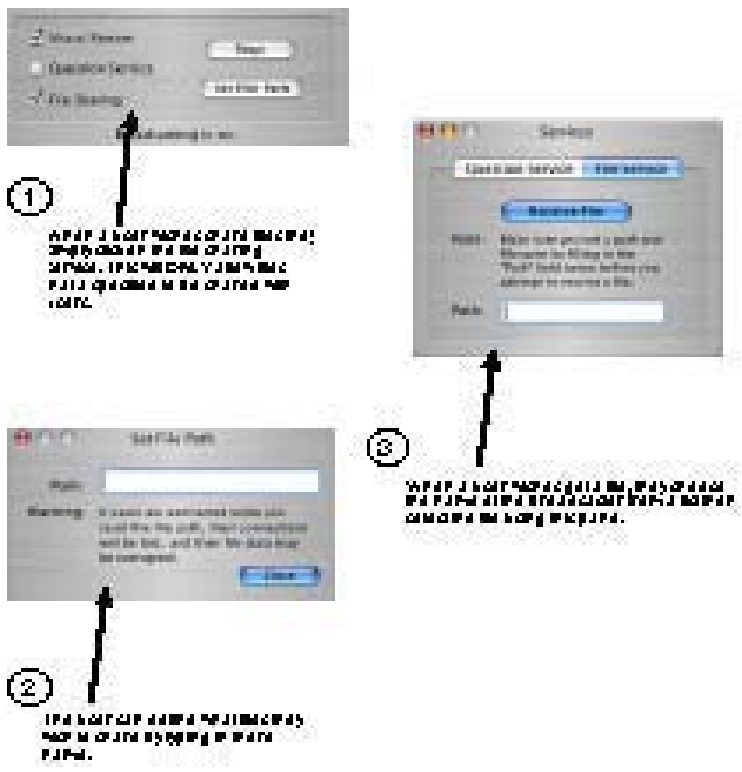

Figure 3: iBroadcast file sharing service

The messaging facility allows clients to communicate with the server. Clients may send messages to the server, which the server user can then view and respond to e.g. by adjusting the presentation. This is particularly useful when dealing with a large audience, such as in a lecture, where a single voice may be difficult to be heard. Students who are 'afraid' of asking questions may also benefit from this feature. Figure 4 shows the question service from both client and server perspectives.

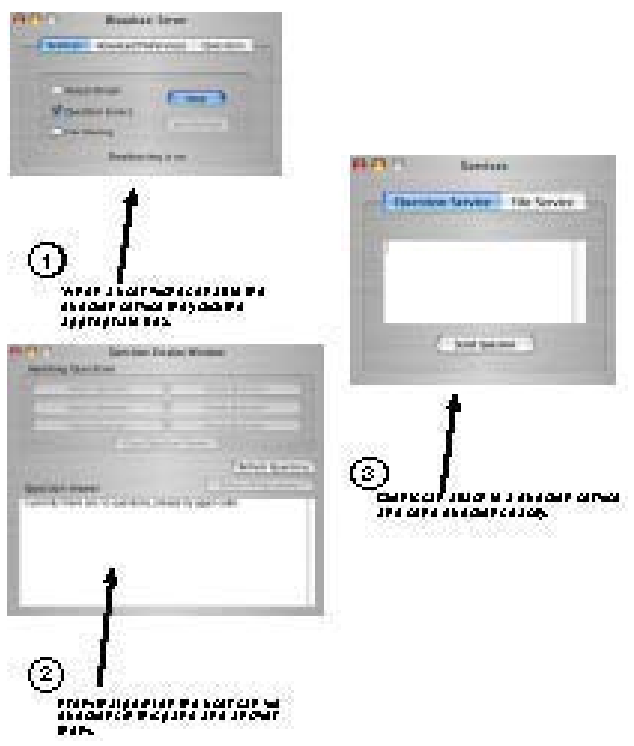

Figure 4: iBroadcast question sharing service 


\subsection{Requirements.}

The prototype environment currently operates on most modern Macintosh computers using the Mac OS X (10.2) or above. While iBroadcast will work on 10/100/1000 MB fixed networks or 11/54 MB wireless networks, the faster networks are recommended to get the best performance from this product.

\section{Related Work.}

A number of different tools exist which provide to varying levels functionality similar to iBroadcast. Traditional network management tools provide mechanisms to share desktops with remote hosts. However, such tools require additional software to be installed and require the user to have an understanding of the underlying network structure. iBroadcast provides similar functionality but hides these additional complexities.

Tools such as iChatAV [5] or iChalk [6] make it possible to have a classroom distributed around the world. However, iBroadcast is designed specifically for teaching and learning environments where information needs to communicated and shared effectively, without the need for technical expertise.

Products such as SnapsX [7] allow presenters to capture events on a screen and record to a file, however they do not support broadcasting to an arbitrary number of clients in real time.

\section{Future Work.}

IBroadcast offers a number of advantages over existing technologies. However there remains a number of areas, which can be improved. These include performance, portability and application.

Future work will focus on improving the underlying algorithms to perform screen capture. It is believed that using compression algorithms can further minimize bandwidth use.

Apple has also released the code for Rendezvous through its Open Source Initiative, allowing developers to port the project to other platforms. To date ports of Rendezvous have emerged for Pocket PC and Linux, and versions of iBroadcast are planned for these platforms.

Future work will also focus on adding a more personal dimension to the software e.g. the provision of aliases such as is possible in ICQ [8]. The ability to identify and relate to people is considered important to the future of this tool, especially if it is applied in a large classroom setting. The messaging environment may change to allow communication between peers in the ad hoc network rather than only directing questions to a central authority. Further applications such as a shared whiteboard may be introduced.

\section{Conclusion}

iBroadcast is a prototype tool that allows the formation of an ad hoc network at any time, and in any location. This tool facilitates more wide spread use of computer supports computer assisted distributed or collaborative learning environments by removing the need for a permanent wired network.

\section{Acknowledgements.}

We would like to thank Jerome Bonnet, Ben Floro, Nicolas Triantafillou and Megan Watson for their efforts in the development of iBroadcast.

\section{References}

[1] Fuller, Anne, McFarlane, Penney,(2002) "Using NetMeeting to Support Offshore Intensive Delivery, Frontiers In Education Proceedings, Boston.

[2] www.uow.edu.au/ dfs/ibroadcast

[3] Apple Computer Developer Connection, "Rendezvous Networking", http://developer.apple.com/macosx/rendezvous/index.html, Last Accessed: $15^{\text {th }}$ February 2004.

[4] Apple Developer Connection, "Inside MacOS X, System Overview”, May 2000 Cupertino, p 29.

[5] Apple Computer Inc, "Apple - Software - iChatAV", http://www.apple.com.au/ichat , Last Accessed $25^{\text {th }}$ February 2004.

[6] Math Game House, "Introducting iChalk", http://www.mathgamehouse.com/ichalk/ , Last Accessed $25^{\text {th }}$ February 2004

[7] Ambrosia "Software, "SnapsX Utility", http://www.ambrosiasw.com/utilities/snapzprox/, Last Accessed $25^{\text {th }}$ February 2004.

[8] ICQ Community Web Page, http://web.icq.com/ , Last Accessed $25^{\text {th }}$ February 2004 